\title{
The Discrete Nonlinear Schrödinger Equation and its Lie Symmetry Reductions
}

\author{
R HERNÁNDEZ HEREDERO ${ }^{\dagger}$ and D LEVI ${ }^{\ddagger}$ \\ † Departamento de Física Teórica II, Facultad de Ciencias Físicas, Universidad \\ Complutense de Madrid, 28040 Madrid, Spain \\ E-mail: rafahh@fis.ucm.es \\ ¥ Dipartimento di Fisica "Edoardo Amaldi", Universitá \\ di Roma Tre and INFN, Sezione di Roma Tre, Via della Vasca Navale 84, \\ 00146 Roma, Italy \\ E-mail: levi@amaldi.fis.uniroma3.it
}

This paper is part of the Proceedings of SIDE V;

Giens, June 21-26, 2002

\begin{abstract}
The Lie algebra $L(h)$ of symmetries of a discrete analogue of the non-linear Schrödinger equation (NLS) is studied. A five-dimensional subspace of $L(h)$, generated by both point and generalized symmetries, transforms into the five-dimensional point symmetry algebra $L(0)$ of the NLS equation. We use the lowest symmetries to do symmetry reduction of the equation, thus obtaining explicit solutions and discrete analogues of elliptic functions.
\end{abstract}

\section{Introduction}

The purpose of this article is to study the Lie algebra $L(h)$ of symmetries of the discrete analogue [1] of the Non-Linear Schrödinger equation (dNLS)

$$
i \dot{Q}_{n}=\frac{1}{h^{2}}\left[2 Q_{n}-\left(1-\epsilon\left|Q_{n}\right|^{2}\right)\left(Q_{n+1}+Q_{n-1}\right)\right],
$$

where $Q_{n}(t)$ is a complex variable, $h$ is an arbitrary constant and $\epsilon$ takes the values \pm 1 .

Among the integrable nonlinear partial differential equations, the non-linear Schrödinger equation (NLS) determines, in the regime of weak nonlinearity, the slow amplitude modulation for a large class of equations [8]. Among all the discretizations of NLS the dNLS plays a distinguished role, being its interesting mathematical properties, such as integrability, what makes studying this equation important.

This article is part of a program, the aim of which is to use integrability theory to describe Lie symmetries of discrete equations and compare them with the results obtained by direct methods. Previous studies have shown [16, 23, 26, 27, 28] that for difference equations on a regular lattice the class of point symmetries is somewhat restricted. More 
interesting symmetries are obtained only if one considers group actions on a finite, or infinite set of points on the lattice $[13,14,25]$ rather than just at one point.

The dNLS equation belongs to the hierarchy of differential-difference equations [9] associated to the discrete Zakharov-Shabat spectral problem

$$
\Phi_{n+1}=\left(Z+Q_{n}(t) I_{1}+R_{n}(t) I_{2}\right) \Phi_{n}
$$

where $\Phi_{n}=\Phi_{n}(t, z)$ is a $2 \times 2$ matrix wavefunction, $Z=\left(\begin{array}{cc}z & 0 \\ 0 & 1 / z\end{array}\right), I_{1}=\left(\begin{array}{ll}0 & 1 \\ 0 & 0\end{array}\right), I_{2}=\left(\begin{array}{ll}0 & 0 \\ 1 & 0\end{array}\right)$, $z$ is the eigenvalue and $Q_{n}(t)$ and $R_{n}(t)$ are two complex scalar functions. Eq. (1.1) is one of the members of the hierarchy of equations associated to the Spectral Problem (1.2) and in the limit $h \rightarrow 0$, setting $Q_{n}(t)=h u(x, t)$ and $x=n h$, it is transformed into the Non-Linear Schrödinger equation (NLS):

$$
i u_{t}+u_{x x}-2 \epsilon|u|^{2} u=0 .
$$

Equation (1.1) is a differential-difference equation that possesses the same integrable structure as the NLS differential equation. It can be described as an integrable discretization of NLS or, better, a semi-discretization, because the time coordinate remains continuous. Eq. (1.1) admits an infinite hierarchy of commuting flows that, in the continuous limit, is transformed into the infinite hierarchy of flows commuting with the NLS equation. This is related to the fact that there are corresponding recursion operators generating both hierarchies. Symmetries and conservation laws of (1.1) have been considered in, for example, [1, 2, 30]. Bäcklund transformations for eq. (1.1) have been considered in $[9]$.

In this article we construct, improving on previous work [15], the Lie algebra of symmetries of the dNLS equation (1.1), and we study its relation to the Lie algebra of point transformations of NLS (1.3). The main objective of the article is the subject of symmetry reduction, that we perform in the dNLS with respect to the most interesting (or simple) symmetries.

In Section 2 we summarize some known results on the hierarchy of the dNLS equation and its integrability, necessary to construct its evolutionary symmetries. Section 3 is devoted to the direct calculation of the Lie point symmetries of the dNLS on an orthogonal lattice, and we show how this direct method yields easily only the simplest intrinsic point symmetries.

In Section 4 we summarize and extend results from [15], writing down explicitly the simplest evolutionary symmetries of dNLS and finding out the structure of the infinite Lie algebra of symmetries. We use the spectral transform to relate the evolutionary symmetries to the linear equations for the evolution of the reflection coefficient. Symmetries acting in the solution space of the evolution equation are transformed into symmetries acting in the space of the spectrum. In the space of the spectrum the commutation relations of the symmetries are easily computed. To conclude the section, we comment on the limiting procedure taking the dNLS equation into the NLS equation. The symmetries are subjected to the same limiting procedure, and this guides us to choose appropriate reductions of the dNLS in the next section.

Section 5 is devoted to the symmetry reduction of the dNLS with respect to the Lie point symmetries and the simplest generalized symmetries. Using this procedure, we are able to obtain similarity solutions of the dNLS. Many results about explicit solutions of 
dNLS are already known, using for example inverse scattering transforms and conservation laws $[31,10]$. We can see how we recover explicit solutions discussed in [10] as solutions of the dNLS reduced with respect to spatial translation. This is the special type of solutions that physically is most reasonable to look for first. Nevertheless, other symmetries allow to search for other type of solutions, which in our case turn out to be defined by ordinary difference equations. This solutions can be considered discrete analogues of the symmetry solutions corresponding to the continuous case. For example, in the case of reduction with respect to time translation, we find what could be a discrete analogue of an elliptic function.

\section{The discrete NLS hierarchy and its integrability}

Let us consider the hierarchy (see $[20,6,9]$ )

$$
\left(\begin{array}{c}
\dot{R_{n}} \\
-\dot{Q_{n}}
\end{array}\right)+\omega\left(L, L^{-1}, t\right)\left(\begin{array}{c}
R_{n} \\
Q_{n}
\end{array}\right)+\tilde{\omega}\left(L, L^{-1}, t\right)(2 n+1)\left(\begin{array}{c}
R_{n} \\
Q_{n}
\end{array}\right)=0
$$

where $\omega\left(L, L^{-1}, t\right)$ and $\tilde{\omega}\left(L, L^{-1}, t\right)$ are entire functions of the recursion operator $L$, defined by

$$
L\left(\begin{array}{l}
A_{n} \\
B_{n}
\end{array}\right)=\left(\begin{array}{c}
A_{n-1}-R_{n-1} a_{n} S_{n}-R_{n} T_{n} \\
B_{n+1}-Q_{n+1} a_{n} S_{n+1}-Q_{n} T_{n+1}
\end{array}\right)
$$

and of its inverse

$$
L^{-1}\left(\begin{array}{c}
A_{n} \\
B_{n}
\end{array}\right)=\left(\begin{array}{c}
A_{n+1}+R_{n+1} a_{n} S_{n+1}+R_{n} Z_{n+1} \\
B_{n-1}+Q_{n-1} a_{n} S_{n}+Q_{n} Z_{n}
\end{array}\right)
$$

with $S_{n}, T_{n}$ and $Z_{n}$ solutions of the inhomogeneous first order equations:

$$
\begin{aligned}
S_{n+1} & =S_{n}-\frac{Q_{n} A_{n}-R_{n} B_{n}}{a_{n}}, \quad a_{n}=1-R_{n} Q_{n} \\
T_{n+1} & =T_{n}-Q_{n} A_{n-1}+R_{n} B_{n+1} \\
Z_{n+1} & =Z_{n}-Q_{n} A_{n+1}+R_{n} B_{n-1} .
\end{aligned}
$$

Whenever $\tilde{\omega}$ is present, the hierarchy (2.1) corresponds to a non-isospectral deformation of the discrete Zakharov and Shabat Spectral Problem (1.2), i. e. the spectral parameter $z$ evolves in time.

For any equation of the hierarchy (2.1) we can write down an explicit evolution equation for the function $\Phi_{n}(t, z)[20,6]$. When the functions $Q_{n}(t), R_{n}(t) S_{n}, T_{n}$ and $Z_{n}$ are asymptotically bounded, i. e. if

$$
\lim _{|n| \rightarrow \infty} Q_{n}(t)=\lim _{|n| \rightarrow \infty} R_{n}(t)=\lim _{|n| \rightarrow \infty} S_{n}=\lim _{|n| \rightarrow \infty} T_{n}=\lim _{|n| \rightarrow \infty} Z_{n}=0
$$

we can then associate to eq. (1.2) a spectrum defined in the complex plane of variable $z$ :

$$
\begin{aligned}
& \mathcal{S}:\left\{\left(\beta^{+}(t, z), \alpha^{+}(z)\right) \quad(|z|>1), \quad\left(\beta^{-}(t, z), \alpha^{-}(z)\right) \quad(|z|<1) ;\right. \\
& \left.z_{j}^{+}\left(\left|z_{j}^{+}\right|>1\right), \quad C_{j}^{+}(t), \quad z_{j}^{-}\left(\left|z_{j}^{-}\right|<1\right), \quad C_{j}^{-}(t) \quad j=1,2, \ldots, N\right\},
\end{aligned}
$$


through the asymptotic behaviour of the solution to the linear problem (1.2):

$$
\begin{array}{ll}
n \rightarrow+\infty: & \Phi_{n}(t, z) \rightarrow\left(\begin{array}{cc}
z^{n} & \beta^{-}(t, z) z^{n} \\
\beta^{+}(t, z) z^{-n} & z^{-n}
\end{array}\right), \\
n \rightarrow-\infty: & \Phi_{n}(t, z) \rightarrow\left(\begin{array}{cc}
\alpha^{+}(z) z^{n} & 0 \\
0 & \alpha^{-}(z) z^{-n}
\end{array}\right) .
\end{array}
$$

The points $z_{j}^{-}$(resp. $z_{j}^{+}$) are isolated inside (resp. outside) the unit disk and $C_{j}^{-}$(resp. $C_{j}^{+}$) are some complex functions of $t$ related to the residues of $\beta^{-}(z, t)$ (resp. $\beta^{+}(z, t)$ ) at the poles $z_{j}^{-}$(resp. $z_{j}^{+}$). When $Q_{n}(t), R_{n}(t), S_{n}, T_{n}$ and $Z_{n}$ satisfy the boundary conditions (2.5), the spectral data $\mathcal{S}$ define the potentials $Q_{n}(t), R_{n}(t)$ in a unique way. There is a one-to-one correspondence between the evolution of the potentials $\left(Q_{n}, R_{n}\right)$ of the discrete Zakharov and Shabat spectral problem (1.2), given by eq. (2.1) and that of the reflection coefficients $\beta^{ \pm}(t, z)$, given by

$$
\frac{d \beta^{ \pm}(t, z)}{d t} \pm \omega\left(z^{2}, z^{-2}, t\right) \beta^{ \pm}(t, z)=0 .
$$

The transmission coefficients $\alpha^{ \pm}(z)$ are constants of the motion. In eq. (2.8) and below, $d / d y$ denotes the total derivative with respect to $y$ and $d z / d t=z \tilde{\omega}\left(z^{2}, 1 / z^{2}, t\right)$.

The hierarchy $(2.1)$ can be reduced setting $R_{n}=\epsilon Q_{n}^{*}$, yielding

$$
\left(\begin{array}{c}
\epsilon \dot{Q}_{n}^{*} \\
-\dot{Q}_{n}
\end{array}\right)+\omega\left(L, L^{-1}, t\right)\left(\begin{array}{c}
\epsilon Q_{n}^{*} \\
Q_{n}
\end{array}\right)+\tilde{\omega}\left(L, L^{-1}, t\right)(2 n+1)\left(\begin{array}{c}
\epsilon Q_{n}^{*} \\
Q_{n}
\end{array}\right)=0
$$

with

$$
\begin{aligned}
& \omega\left(z^{2}, z^{-2}, t\right)=\omega_{1}\left(z^{2}, t\right)-\omega_{1}^{*}\left(z^{*-2}, t\right), \\
& \tilde{\omega}\left(z^{2}, z^{-2}, t\right)=\tilde{\omega}_{1}\left(z^{2}, t\right)-\tilde{\omega}_{1}^{*}\left(z^{*-2}, t\right),
\end{aligned}
$$

where $\omega_{1}$, and $\tilde{\omega}_{1}$ are entire functions of their first argument, and the star ${ }^{*}$ denotes complex conjugation. Under this reduction the spectrum $\mathcal{S}$ can be defined in terms of a single function as the following relation between the spectral parameters holds [20, 9]:

$$
\begin{aligned}
& \beta^{+}(t, z)=-\epsilon\left[\beta^{-}\left(t, \frac{1}{z^{*}}\right)\right]^{*}, \\
& z_{j}^{+}=\left(\frac{1}{z_{j}^{-}}\right)^{*}, \\
& C_{j}^{+}(t)=\epsilon\left(z_{j}^{+}\right)^{2}\left(C_{j}^{-}(t)\right)^{*} .
\end{aligned}
$$

From now on we will denote $\beta=\beta^{-}$, and its evolution is given by

$$
\frac{d \beta}{d t}=\omega\left(z^{2}, z^{-2}, t\right) \beta, \quad \frac{d z}{d t}=z \tilde{\omega}\left(z^{2}, z^{-2}, t\right) .
$$

As examples of nonlinear equations, let us consider at first the case when $\omega_{1}\left(z^{2}, t\right)=$ $\alpha_{0}+\alpha_{1} z^{2}+\alpha_{2} z^{4}\left(\alpha_{j}, j=0,1,2\right.$ constants $)$ and $\tilde{\omega}_{1}\left(z^{2}, t\right)=0$, i. e. an isospectral deformation of the discrete Spectral Problem (1.2). In this case the nonlinear evolution equation reads

$$
\begin{aligned}
& \dot{Q}_{n}=(\left.\alpha_{0}-\alpha_{0}^{*}\right) Q_{n}+\left(1-\epsilon\left|Q_{n}\right|^{2}\right)\left(\alpha_{1} Q_{n+1}-\alpha_{1}^{*} Q_{n-1}\right)+\left(1-\epsilon\left|Q_{n}\right|^{2}\right) . \\
& \cdot\left\{\alpha_{2}\left[Q_{n+2}\left(1-\epsilon\left|Q_{n+1}\right|^{2}\right)-\epsilon Q_{n+1}\left(Q_{n}^{*} Q_{n+1}+Q_{n} Q_{n-1}^{*}\right)\right]\right. \\
&\left.-\alpha_{2}^{*}\left[Q_{n-2}\left(1-\epsilon\left|Q_{n-1}\right|^{2}\right)-\epsilon Q_{n-1}\left(Q_{n}^{*} Q_{n-1}+Q_{n} Q_{n+1}^{*}\right)\right]\right\} .
\end{aligned}
$$


In correspondence with eq. (2.15) $\beta$ evolves according to the equation:

$$
\frac{d \beta}{d t}+\left[\alpha_{0}-\alpha_{0}^{*}+\alpha_{1} z^{2}-\alpha_{1}^{*} \frac{1}{z^{2}}+\alpha_{2} z^{4}-\alpha_{2}^{*} \frac{1}{z^{4}}\right] \beta=0 \quad \frac{d z}{d t}=0
$$

The dNLS (1.1) is obtained from (2.15) by choosing $\alpha_{0}=-\alpha_{1}=i / h^{2}$ and $\alpha_{2}=0$ and the time evolution of its spectrum is given by:

$$
\frac{d \beta}{d t}=\frac{i}{h^{2}}\left[z^{2}+\frac{1}{z^{2}}-2\right] \beta ; \quad \frac{d z}{d t}=0 .
$$

As a complementary example, let us consider a non-isospectral ( $n$-dependent) equation obtained by considering $\omega_{1}\left(z^{2}, t\right)=0$ and $\tilde{\omega}_{1}\left(z^{2}, t\right)=\tilde{\alpha}_{0}+\tilde{\alpha}_{1} z^{2}$. In this case we get:

$$
\begin{gathered}
\dot{Q}_{n}=\left(\tilde{\alpha}_{0}-\tilde{\alpha}_{0}^{*}\right)(2 n+1) Q_{n}+\tilde{\alpha}_{1}\left[(2 n+3)\left(1-\epsilon\left|Q_{n}\right|^{2}\right) Q_{n+1}+2 \epsilon Q_{n} S_{n}^{*}\right] \\
+\tilde{\alpha}_{1}^{*}\left[(2 n-1)\left(1-\epsilon\left|Q_{n}\right|^{2}\right) Q_{n-1}+2 \epsilon Q_{n} S_{n}\right]
\end{gathered}
$$

where

$$
S_{n+1}-S_{n}=-Q_{n} Q_{n+1}^{*}
$$

and correspondingly $\beta$ satisfies the following equation:

$$
\frac{d \beta}{d t}=0 ; \quad \frac{d z}{d t}=z\left[\tilde{\alpha}_{0}-\tilde{\alpha}_{0}^{*}+\tilde{\alpha}_{1} z^{2}-\tilde{\alpha}_{1}^{*} \frac{1}{z^{2}}\right] .
$$

\section{Direct calculation of symmetries of the discrete NLS}

In this Section, we calculate directly the point symmetries of dNLS (1.1), using the theory described in [19]. Decomposing $Q_{n}$ in real and imaginary parts $Q_{n}=u_{n}+i v_{n}$, dNLS becomes

$$
\begin{aligned}
\frac{d u_{n}}{d t} & =\frac{1}{h^{2}}\left\{2 v_{n}-\left[1-\epsilon\left(u_{n}^{2}+v_{n}^{2}\right)\right]\left(v_{n+1}+v_{n-1}\right)\right\} \\
\frac{d v_{n}}{d t} & =\frac{1}{h^{2}}\left\{\left[1-\epsilon\left(u_{n}^{2}+v_{n}^{2}\right)\right]\left(u_{n+1}+u_{n-1}\right)-2 u_{n}\right\} .
\end{aligned}
$$

In eq. (3.1) the dependent variables $\left(u_{n}(t), v_{n}(t)\right)$ are defined on the space of the independent variables $\left(x_{n}, t\right)$ where $x_{n}$ defines the points of a lattice while $t$ is a continuous "time". It is convenient to characterize each point in the space of the independent variables by two indices, say $m, n$, where $m$ parametrizes the "time" while $n$ characterizes the position in the lattice $\left(x_{n, m}, t_{n, m}\right)$. In such a way eq. (3.1) reads:

$$
\begin{aligned}
& \frac{d u_{n, m}}{d t}=\frac{1}{h^{2}}\left\{2 v_{n, m}-\left[1-\epsilon\left(u_{n, m}^{2}+v_{n, m}^{2}\right)\right]\left(v_{n+1, m}+v_{n-1, m}\right)\right\} \\
& \frac{d v_{n, m}}{d t}=\frac{1}{h^{2}}\left\{\left[1-\epsilon\left(u_{n, m}^{2}+v_{n, m}^{2}\right)\right]\left(u_{n+1, m}+u_{n-1, m}\right)-2 u_{n, m}\right\} .
\end{aligned}
$$

The postulated generic generator of the point symmetry at the point $(n, m)$ is given by

$$
\hat{\mathcal{X}}_{n, m}=\xi_{n, m} \partial_{x_{n, m}}+\tau_{n, m} \partial_{t_{n, m}}+\phi_{n, m}^{1} \partial_{u_{n, m}}+\phi_{n, m}^{2} \partial_{v_{n, m}}
$$


where $\xi_{n, m}, \tau_{n, m}, \phi_{n, m}^{1}$ and $\phi_{n, m}^{2}$, are functions of $\left(x_{n, m}, t_{n, m}, u_{n, m}, v_{n . m}\right)$. The prolongation of $\hat{\mathcal{X}}_{n, m}$ is given by

$$
\operatorname{pr} \hat{\mathcal{X}}_{n, m}=\sum_{n, m} \hat{\mathcal{X}}_{n, m}+\phi_{n, m}^{1, t_{n, m}} \partial_{\dot{u}_{n, m}}+\phi_{n, m}^{2, t_{n, m}} \partial_{\dot{v}_{n, m}}
$$

where $\phi_{n, m}^{1, t}$ and $\phi_{n, m}^{2, t}$ are given by

$$
\begin{aligned}
& \phi_{n, m}^{1, t_{n, m}}=D_{t_{n, m}} \phi_{n, m}^{1}-\dot{u}_{n, m} D_{t_{n, m}} \tau_{n, m}-u_{n, m, x_{n, m}} D_{t_{n, m}} \xi_{n, m} \\
& \phi_{n, m}^{2, t_{n, m}}=D_{t_{n, m}} \phi_{n, m}^{2}-\dot{v}_{n, m} D_{t_{n, m}} \tau_{n, m}-v_{n, m, x_{n, m}} D_{t_{n, m}} \xi_{n, m} .
\end{aligned}
$$

and the sum is extended to all points of the lattice present in the equation.

We choose a lattice given by the following equations:

$$
\begin{gathered}
x_{n+1, m}-x_{n, m}=h \\
x_{n, m+1}=x_{n, m} \\
t_{n+1, m}=t_{n, m} .
\end{gathered}
$$

Application of the prolonged generator $(3.3)$ to equations $(3.2,3.5)$ gives the defining equations for the symmetry, that must hold on solutions of $(3.2,3.5)$. The system of equations obtained by applying (3.3) to the lattice (3.5) implies that $\xi_{n, m}$ is constant and $\tau_{n, m}=\tau_{n, m}\left(t_{n, m}\right)$. Applying eq. (3.3) to the dNLS we obtain that there are only three independent intrinsic point symmetries:

$$
\hat{\mathcal{X}}_{n, m}=a \partial_{x_{n, m}}+b \partial_{t_{n, m}}+c\left(v_{n, m} \partial_{u_{n, m}}-u_{n, m} \partial_{v_{n, m}}\right)
$$

which, going back to the notation of eq. (1.1) read:

$$
\hat{\mathcal{X}}_{1}=Q_{n} \partial_{Q_{n}}-Q_{n}^{*} \partial_{Q_{n}^{*}} ; \quad \hat{\mathcal{X}}_{2}=\partial_{n} ; \quad \hat{\mathcal{X}}_{3}=\partial_{t} .
$$

Note that the form of the two last symmetries, those involving just independent variables, depends strictly on the form of the lattice (3.5). Selecting other type of lattice would change $\mathcal{X}_{2}$ and $\mathcal{X}_{3}$.

\section{Symmetries of the discrete NLS}

Symmetries of the dNLS can be constructed by considering flows

$$
\frac{d}{d \lambda} Q_{n}=f\left(t, n, Q_{n}, Q_{n+1}, Q_{n-1}, \ldots\right)
$$

commuting with (1.1). Due to the one-to-one correspondence between the evolution of $Q_{n}$ and that of the reflection coefficient $\beta$, commuting flows acting in the solution space of the evolution equation have counterparts in the form of symmetries acting in the space of the reflection coefficient. Thus we can define the symmetries by looking for commuting flows among the possible evolutions (2.14) of the reflection coefficients $\beta(t, z, \lambda)$

$$
\frac{d}{d \lambda} \beta(t, z, \lambda)=\omega\left(z^{2}, z^{-2}, t\right) \beta(t, z, \lambda), \quad \frac{d}{d \lambda} z(t, \lambda)=z \tilde{\omega}\left(z^{2}, z^{-2}, t\right)
$$


with those of the dNLS (2.17). From this commutation we get:

$$
\omega\left(z^{2}, z^{-2}, t\right)=-\frac{2 i t}{h^{2}}\left(z^{2}-z^{-2}\right) \tilde{\omega}\left(z^{2}, z^{-2}, t\right)+\bar{\omega}\left(z^{2}, z^{-2}\right), \quad \frac{d \tilde{\omega}}{d t}=0
$$

where $\bar{\omega}$ is an arbitrary entire function of $z^{2}$ and $z^{-2}$ of the form (2.10) and $\tilde{\omega}$ is constrained so that $\omega$ is of the form (2.10).

We can distinguish various types of symmetries, corresponding to isospectral or nonisospectral flows. In the isospectral case we have that $\tilde{\omega}=0$ and then from eqs. (4.3), (2.10) we get that $\omega$ is given in terms of an arbitrary entire function of $z^{2}$ and $z^{-2}$. In this case we can define a base of symmetry generators in the spectral space as:

$$
X_{0}=\beta \partial_{\beta}, \quad X_{j}^{R}=\left(z^{2 j}-z^{-2 j}\right) \beta \partial_{\beta}, \quad X_{j}^{I}=i\left(z^{2 j}+z^{-2 j}\right) \beta \partial_{\beta} .
$$

Considering complex linear combinations of $X_{j}^{R}$ and $X_{j}^{I}$, we can redefine the base (4.4) as

$$
X_{j}=z^{2 j} \beta \partial_{\beta},
$$

with $j$ an arbitrary integer.

In the non-isospectral case, $\tilde{\omega} \neq 0$ and $\bar{\omega}=0$, and in an analogous way to the isospectral case we can choose the following base of symmetry generators:

$$
Z_{j}=z^{2 j}\left[\frac{2 i t}{h^{2}}\left(z^{2}-z^{-2}\right) \beta-z \beta_{z}\right] \partial_{\beta} .
$$

We present explicitly the simplest symmetries of the dNLS, which we will need later:

$$
\begin{aligned}
& X_{0}=Q_{n} \partial_{Q_{n}}-Q_{n}^{*} \partial_{Q_{n}^{*}} \\
& X_{1}=\left(1-\epsilon\left|Q_{n}\right|^{2}\right) Q_{n+1} \partial_{Q_{n}}-\left(1-\epsilon\left|Q_{n}\right|^{2}\right) Q_{n-1}^{*} \partial_{Q_{n}^{*}} \\
& X_{-1}=\left(1-\epsilon\left|Q_{n}\right|^{2}\right) Q_{n-1} \partial_{Q_{n}}-\left(1-\epsilon\left|Q_{n}\right|^{2}\right) Q_{n+1}^{*} \partial_{Q_{n}^{*}} \\
& X_{2}=\left(1-\epsilon\left|Q_{n}\right|^{2}\right)\left[Q_{n+2}\left(1-\epsilon\left|Q_{n+1}\right|^{2}\right)-\epsilon Q_{n+1}\left(Q_{n}^{*} Q_{n+1}+Q_{n-1}^{*} Q_{n}\right)\right] \partial_{Q_{n}} \\
& -\left(1-\epsilon\left|Q_{n}\right|^{2}\right)\left[Q_{n-2}^{*}\left(1-\epsilon\left|Q_{n-1}\right|^{2}\right)-\epsilon Q_{n-1}^{*}\left(Q_{n}^{*} Q_{n+1}+Q_{n-1}^{*} Q_{n}\right)\right] \partial_{Q_{n}^{*}} \\
& X_{-2}=\left(1-\epsilon\left|Q_{n}\right|^{2}\right)\left[Q_{n-2}\left(1-\epsilon\left|Q_{n-1}\right|^{2}\right)-\epsilon Q_{n-1}\left(Q_{n}^{*} Q_{n-1}+Q_{n+1}^{*} Q_{n}\right)\right] \partial_{Q_{n}} \\
& -\left(1-\epsilon\left|Q_{n}\right|^{2}\right)\left[Q_{n+2}^{*}\left(1-\epsilon\left|Q_{n+1}\right|^{2}\right)-\epsilon Q_{n+1}^{*}\left(Q_{n} Q_{n+1}^{*}+Q_{n-1} Q_{n}^{*}\right)\right] \partial_{Q_{n}^{*}} \\
& Z_{0}=\left[-\frac{2 i t}{h^{2}}\left(1-\epsilon\left|Q_{n}\right|^{2}\right)\left(Q_{n+1}-Q_{n-1}\right)-(2 n+1) Q_{n}\right] \partial_{Q_{n}} \\
& +\left[-\frac{2 i t}{h^{2}}\left(1-\epsilon\left|Q_{n}\right|^{2}\right)\left(Q_{n+1}^{*}-Q_{n-1}^{*}\right)+(2 n+1) Q_{n}^{*}\right] \partial_{Q_{n}^{*}} \\
& Z_{1}=\left\{\frac{2 i t}{h^{2}}\left(1-\epsilon\left|Q_{n}\right|^{2}\right)\left[Q_{n+2}-\epsilon\left(Q_{n+1}^{*} Q_{n+2}+Q_{n}^{*} Q_{n+1}+Q_{n-1}^{*} Q_{n}\right) Q_{n+1}\right]\right. \\
& \left.-\frac{2 i t}{h^{2}} Q_{n}+(2 n+3)\left(1-\epsilon\left|Q_{n}\right|^{2}\right) Q_{n+1}+2 \epsilon Q_{n} S_{n}^{*}\right\} \partial_{Q_{n}} \\
& +\left\{-\frac{2 i t}{h^{2}}\left(1-\epsilon\left|Q_{n}\right|^{2}\right)\left[Q_{n-2}^{*}-\epsilon\left(Q_{n-1} Q_{n-2}^{*}+Q_{n} Q_{n-1}^{*}+Q_{n+1} Q_{n}^{*}\right) Q_{n-1}^{*}\right]\right. \\
& \left.+\frac{2 i t}{h^{2}} Q_{n}^{*}-(2 n-1)\left(1-\epsilon\left|Q_{n}\right|^{2}\right) Q_{n-1}^{*}-2 \epsilon Q_{n}^{*} S_{n}^{*}\right\} \partial_{Q_{n}^{*}}
\end{aligned}
$$




$$
\begin{aligned}
Z_{-1}= & \left\{-\frac{2 i t}{h^{2}}\left(1-\epsilon\left|Q_{n}\right|^{2}\right)\left[Q_{n-2}-\epsilon\left(Q_{n-1}^{*} Q_{n-2}+Q_{n}^{*} Q_{n-1}+Q_{n+1}^{*} Q_{n}\right) Q_{n-1}\right]\right. \\
& \left.+\frac{2 i t}{h^{2}} Q_{n}+(2 n-1)\left(1-\epsilon\left|Q_{n}\right|^{2}\right) Q_{n-1}+2 \epsilon Q_{n} S_{n}\right\} \partial_{Q_{n}} \\
+ & \left\{\frac{2 i t}{h^{2}}\left(1-\epsilon\left|Q_{n}\right|^{2}\right)\left[Q_{n+2}^{*}-\epsilon\left(Q_{n+1} Q_{n-+}^{*}+Q_{n} Q_{n+1}^{*}+Q_{n-1} Q_{n}^{*}\right) Q_{n+1}^{*}\right]\right. \\
& \left.-\frac{2 i t}{h^{2}} Q_{n}^{*}-(2 n+3)\left(1-\epsilon\left|Q_{n}\right|^{2}\right) Q_{n+1}^{*}-2 \epsilon Q_{n}^{*} S_{n}\right\} \partial_{Q_{n}^{*}}
\end{aligned}
$$

where the function $S_{n}$ appearing in symmetries $Z_{1}$ and $Z_{-1}$ was defined in (2.18). Taking into account the temporal evolution of dNLS (1.1), $S_{n}$ can be characterized as the function satisfying the system

$$
S_{n+1}=S_{n}-Q_{n} Q_{n+1}^{*}, \quad \dot{S}_{n}=\frac{i}{h^{2}}\left[\left|Q_{n}\right|^{2}-\left(1-\epsilon\left|Q_{n}\right|^{2}\right) Q_{n-1}^{*} Q_{n+1}\right] .
$$

Note that $X_{0}$ is a point symmetry, and only one additional independent point symmetry can be obtained, $2 X_{0}-X_{1}-X_{-1}=\dot{Q}_{n} \partial_{Q_{n}}-\dot{Q}_{n}^{*} \partial_{Q_{n}^{*}}$. The remaining independent symmetries are generalized symmetries, and $Z_{i}$ with $|i| \geq 1$ are non-local symmetries. The lattice point symmetry $\hat{\mathcal{X}}_{2}=\partial_{n}$ in (3.7) cannot be retrieved by a symmetry (4.1) depending only on a finite number of fields $Q_{i}$.

The structure of the algebra $\mathcal{L}$ of infinitesimal symmetries of dNLS can be inferred from the commutation relations

$$
\begin{aligned}
& {\left[X_{i}, X_{j}\right]=0, \quad\left[X_{i}, Z_{j}\right]=-2 i X_{i+j}} \\
& {\left[Z_{i}, Z_{j}\right]=-2(i-j) Z_{i+j} .}
\end{aligned}
$$

The subalgebra $\mathcal{L}_{1}$ generated by $X_{i}, i \in \mathbf{Z}$, is abelian. The symmetries $Z_{i}, i \in \mathbf{Z}$ also generate a subalgebra $\mathcal{L}_{0}$, which is perfect, i. e. $\left[\mathcal{L}_{0}, \mathcal{L}_{0}\right]=\mathcal{L}_{0}$. The structure of the whole algebra is that of of semidirect sum

$$
\mathcal{L}=\mathcal{L}_{0} \rightrightarrows \mathcal{L}_{1}
$$

\subsection{Continuous limit}

The continuous limit

$$
Q_{n}(t)=h u(x, t), \quad x=n h
$$

transforms the dNLS equation (1.1) into the NLS equation (1.3)

$$
i u_{t}+u_{x x}-2 \epsilon|u|^{2} u=O\left(h^{2}\right)
$$

Here we study the relationship between the symmetries of (1.1) and those of its continuous limit (1.3). Particularly, we are interested in finding precursors of the point symmetries of NLS in the symmetry algebra of (1.1). We will consider the point symmetries of NLS:

$$
\begin{aligned}
& y_{1}=u \partial_{u}-u^{*} \partial_{u^{*}}, \\
& y_{2}=u_{x} \partial_{u}+u_{x}^{*} \partial_{u^{*}}, \\
& y_{3}=u_{t} \partial_{u}+u_{t}^{*} \partial_{u^{*}}, \\
& y_{4}=\left(i x u-2 t u_{x}\right) \partial_{u}-\left(i x u^{*}+2 t u_{x}^{*}\right) \partial_{u^{*}}, \\
& y_{5}=\left(u+2 t u_{t}+x u_{x}\right) \partial_{u}+\left(u^{*}+2 t u_{t}^{*}+x u_{x}^{*}\right) \partial_{u^{*}} .
\end{aligned}
$$


This is a five-dimensional algebra, while we have seen that the point symmetry subalgebra of dNLS (1.1) is three-dimensional, generated by the vector fields $X_{0}$ and $2 X_{0}-X_{1}-$ $X_{-1}$ and the lattice symmetry $\hat{\mathcal{X}}_{2}=\partial_{n}$ (cf. (3.7)). The remaining two independent point symmetries of NLS must be recovered from the continuous limit of some generalized symmetry of (1.1). The lattice symmetry $\hat{\mathcal{X}}_{2}$ can also be approximated in the continuous limit by a generalized symmetry of (1.1).

Taking into account that under the transformation (4.8), the function $S$ appearing in (4.7) reduces to

$$
S \rightarrow-h \int|u|^{2} d x+h^{2} \int\left(u_{x} u^{*}-u u_{x}^{*}\right) d x+O\left(h^{3}\right),
$$

the appropriate combinations of the symmetries, such that in the continuous limit we get (4.9), are

$$
\begin{aligned}
& Y_{1} \equiv X_{0}=y_{1}, \quad Y_{2} \equiv \frac{X_{1}-X_{0}}{h}=y_{2}+O(h) \\
& Y_{3} \equiv \frac{i}{h^{2}}\left(X_{-1}-2 X_{0}+X_{1}\right)=y_{3}+O\left(h^{2}\right) \\
& Y_{4} \equiv-h i \frac{Z_{0}+X_{0}}{2}=y_{4}+O\left(h^{2}\right), \quad Y_{5} \equiv \frac{Z_{1}-Z_{-1}}{4}=y_{5}+O(h)
\end{aligned}
$$

The point symmetries $Y_{1}, Y_{3}$ of the dNLS equation produce, in the continuous limit, the point symmetries $y_{1}, y_{3}$ of NLS. NLS point symmetries $y_{2}, y_{4}$ and $y_{5}$ have to be recovered from non-point symmetries $Y_{2}, Y_{4}$ and $Y_{5}$ of dNLS (i. e. $X_{1}, Z_{0}, Z_{1}, Z_{-1}$ besides $X_{0}$ ).

\begin{tabular}{|c|c|c|c|c|c|}
\hline & $Y_{4}$ & $Y_{5}$ & & $y_{4}$ & $y_{5}$ \\
\hline$Y_{2}$ & $-i Y_{1}-h i Y_{2}$ & $-Y_{2}+\frac{h i}{2} Y_{3}-\frac{h^{2}}{2} Y_{6}$ & $y_{2}$ & $-i y_{1}$ & $-y_{2}$ \\
\hline$Y_{3}$ & $2 Y_{2}+h i Y_{3}$ & $-2 Y_{3}-\frac{h^{2}}{2} i Y_{7}$ & $y_{3}$ & $2 y_{2}$ & $-2 y_{3}$ \\
\hline$Y_{4}$ & 0 & $Y_{4}+\frac{h}{2} i Y_{1}+\frac{h^{2}}{2} Y_{8}-\frac{h^{3}}{2} Y_{3}$ & $y_{4}$ & 0 & $y_{4}$ \\
\hline$Y_{5}$ & $-\left[Y_{4}, Y_{5}\right]$ & $\begin{array}{lll}2 & 0 & 2\end{array}$ & $y_{5}$ & $-y_{4}$ & 0 \\
\hline
\end{tabular}
Thus, a contraction of the Lie algebra of symmetries of dNLS is occurring [16].

The non-zero elements of the commutator tables for the symmetries of (1.1) and for the NLS symmetries are, respectively

where $Y_{6} \equiv\left(X_{2}+3 X_{0}-3 X_{1}-X_{-1}\right) / h^{3}, Y_{7} \equiv\left(X_{-2}+6 X_{0}-4 X_{1}-4 X_{-1}+X_{2}\right) / h^{4}$ and $Y_{8} \equiv i\left(Z_{1}+Z_{-1}+2 Z_{0}-2 X_{-1}-2 X_{1}+4 X_{0}\right) /(2 h)$ are combinations with well defined continuous limit. We can see from the previous tables how the point-symmetry subalgebra of (1.3), generated by $y_{1}, y_{2}, y_{3}, y_{4}$ and $y_{5}$, is the image under a contraction of the set generated by $Y_{1}, Y_{2}, Y_{3}, Y_{4}$ and $Y_{5}$. This set contains the subalgebra of point algebras of (1.1), but it is not an algebra itself.

\section{$5 \quad$ Symmetry reductions}

In this Section we present the results obtained by carrying out the symmetry reduction of the dNLS with respect to the Lie point symmetries and the simplest generalized symmetries. For completeness we first present the corresponding results on the continuous NLS equation. 


\subsection{Continuous case}

It is convenient now to rewrite the Lie point symmetries (4.9) of the NLS equation (1.3) using the polar representation of the complex function $u(x, t)$, i. e. $u(x, t)=\rho(x, t) e^{i \phi(x, t)}$ :

$$
\begin{gathered}
y_{1}=-i \partial_{\phi}, \quad y_{2}=\rho_{x} \partial_{\rho}+\phi_{x} \partial_{\phi}, \quad y_{3}=\rho_{t} \partial_{\rho}+\phi_{t} \partial_{\phi}, \\
y_{4}=-2 t \partial_{x}+x \partial_{\phi}, \quad y_{5}=x \partial_{x}+2 t \partial_{t}-\rho \partial_{\rho} .
\end{gathered}
$$

The symmetry reductions are as follows.

- In the case of $y_{2}$ the NLS equation (1.3) reduces to

$$
\rho_{t}=0 ; \quad \phi_{t}=-2 \epsilon \rho^{2},
$$

which can be easily solved and give $u(x, t)=\rho_{0} e^{i\left(\phi_{0}+2 \epsilon \rho_{0}^{2} t\right)}, \rho_{0}$ and $\phi_{0}$ being arbitrary integration constants.

- In the case of $y_{3}$ the NLS equation (1.3) reduces to

$$
2 \rho_{x} \phi_{x}+\rho \phi_{x x}=0 ; \quad \rho_{x x}=\rho \phi_{x}^{2}+2 \epsilon \rho^{3}
$$

which can be reduced to the following elliptic equation for the variable $v=\rho^{2}$

$$
v_{x}=2 \sqrt{\epsilon v^{3}+K_{1} v-K_{2}^{2}}
$$

where $K_{1}$ and $K_{2}$ are integration constants and $\phi$ is such that $\phi_{x}=K_{2} / v$.

- In the case of $y_{4}$ the invariant variables are:

$$
\rho(x, t)=\rho_{0}(t) ; \quad \phi(x, t)=\phi_{0}(t)-\frac{x^{2}}{4 t}
$$

and the NLS equation (1.3) is solved by $u(x, t)=K_{1} / \sqrt{t} \cdot \exp \left(i\left(K_{2}-2 \epsilon K_{1}^{2} \log (t)-x^{2} / 4 t\right)\right)$, being $K_{1}$ and $K_{2}$ integration constants.

- Finally in the case of $y_{5}$ the invariant variables are:

$$
\rho(x, t)=\frac{\rho_{0}(\eta)}{\sqrt{t}} ; \quad \phi(x, t)=\phi_{0}(\eta) ; \quad \eta=\frac{x}{\sqrt{t}}
$$

In terms of these variables the NLS equation (1.3) reduces to:

$$
\begin{aligned}
& \rho_{0}+\eta \rho_{0, \eta}+4 \rho_{0, \eta} \phi_{0, \eta}+2 \rho_{0} \phi_{0, \eta \eta}=0 \\
& \rho_{0, \eta \eta}-\frac{1}{2} \eta \rho_{0} \phi_{0, \eta}-\rho_{0} \phi_{0, \eta}^{2}-2 \epsilon \rho_{0}^{3}=0 .
\end{aligned}
$$

Defining the new variable $Y(\eta)$ so that $\phi_{0, \eta}=-\frac{1}{4}\left(\eta+\frac{Y}{Y_{\eta}}\right)$ and $\rho_{0}^{2}=Y_{\eta}$, eq. (5.7) is identically satisfied and eq. (5.8) reduces to

$$
Y_{\eta \eta}^{2}+4\left(\eta Y_{\eta}-Y\right)^{2}-16 \epsilon Y_{\eta}^{3}-4 \epsilon \mu^{2} Y_{\eta}=0
$$


where $\mu$ is an arbitrary integration constant. For $\epsilon=-1$ it can be shown [4] that it has the only solution $Y=0$ while for $\epsilon=1$ eq. (5.9) can be reduced to the Painlevé IV equation [17]

$$
W W_{\eta \eta}=\frac{1}{2} W_{\eta}^{2}-6 W^{4}+8 \eta W^{3}-2 \eta^{2} W^{2}-\frac{1}{2}(\mu-1)^{2},
$$

by defining:

$$
Y=\frac{1}{2} W(W-\eta)^{2}+\frac{1}{8 W}\left(W_{\eta}^{2}-2 W_{\eta}-\mu^{2}+1\right)
$$

\subsection{Discrete case}

The symmetries that we will use to find reductions of dNLS are $Y_{2}, Y_{3}, Y_{4}$, while in the case of $Y_{5}$ the constraint is given by a nonlocal fifth order nonlinear difference system of equations which is too involved to get a significant explicit reduction.

Reduction by $\mathbf{Y}_{\mathbf{2}}$ The reduction is obtained by solving the following equation:

$$
\left(1-\epsilon\left|Q_{n}\right|^{2}\right)\left(Q_{n+1}-Q_{n-1}\right)=0
$$

One solution is given by $Q_{n+1}=Q_{n-1}$, i. e.

$$
Q_{n}=\alpha(t)+(-1)^{n} \beta(t)
$$

Writing this solution as $Q_{2 n}=\tilde{a}(t), Q_{2 n+1}=\tilde{b}(t)$ and substituting it into (1.1), we get that $\tilde{a}$ and $\tilde{b}$ must satisfy the following equations

$$
\begin{aligned}
i \dot{\tilde{a}} & =\frac{2}{h^{2}}\left[\tilde{a}-\left(1-\epsilon|\tilde{a}|^{2}\right) \tilde{b}\right] \\
i \dot{\tilde{b}} & =\frac{2}{h^{2}}\left[\tilde{b}-\left(1-\epsilon|\tilde{b}|^{2}\right) \tilde{a}\right]
\end{aligned}
$$

Defining $\tilde{a}=\exp \left(-\frac{2 i}{h^{2}} t\right) a(t)$ and $\tilde{b}=\exp \left(-\frac{2 i}{h^{2}} t\right) b(t)$ we get

$$
i \dot{a}+\frac{2}{h^{2}}\left(1-\epsilon|a|^{2}\right) b=0, \quad i \dot{b}+\frac{2}{h^{2}}\left(1-\epsilon|b|^{2}\right) a=0 .
$$

Eqs. (5.12) can be solved in terms of an elliptic integral

$$
\dot{B}=-\frac{1}{h^{2}}\left(B^{4}+8\left(\epsilon K_{0}-2\right) B^{2}+K_{1}\right)^{\frac{1}{2}}
$$

where $K_{0}$ and $K_{1}$ are two integration constants and, in polar coordinates $a=\rho_{a} \exp \left(i \phi_{a}\right)$, $b=\rho_{b} \exp \left(i \phi_{b}\right)$, the function $B$ is defined by $B=\rho_{b} \rho_{a} \sin \left(\phi_{a}-\phi_{b}\right)$. In terms of the elliptic function $B$

$$
\begin{aligned}
& \rho_{a}^{2}=\frac{1}{8} \epsilon B^{2}-\frac{h^{2}}{8} \dot{B}+\frac{K_{0}}{2}, \\
& \rho_{b}^{2}=\frac{1}{8} \epsilon B^{2}+\frac{h^{2}}{8} \dot{B}+\frac{K_{0}}{2}
\end{aligned}
$$


and the phases $\phi_{a}$ and $\phi_{b}$ are obtained by quadrature from the following equations:

$$
\begin{aligned}
& \dot{\phi}_{a}=\frac{\dot{\rho}_{a}}{\rho_{a}} \frac{\left(\rho_{a}^{2} \rho_{b}^{2}-B^{2}\right)^{\frac{1}{2}}}{B}, \\
& \dot{\phi}_{b}=-\frac{\dot{\rho}_{b}}{\rho_{b}} \frac{\left(\rho_{a}^{2} \rho_{b}^{2}-B^{2}\right)^{\frac{1}{2}}}{B} .
\end{aligned}
$$

Realizing that eq. (5.12) admits two conserved quantities

$$
\begin{aligned}
& \left(1-\epsilon \rho_{a}^{2}\right)\left(1-\epsilon \rho_{b}^{2}\right)=K, \\
& \rho_{a} \rho_{b} \cos \left(\phi_{b}-\phi_{a}\right)=C
\end{aligned}
$$

we can give an alternative description of the solutions, readily interpretable in terms of a non-linear two-body chain [18]. One can parametrize the algebraic curve given by (5.14) in terms of elliptic functions of a parameter $\theta, \rho_{a}=\mathrm{s}(\theta), \rho_{b}=\mathrm{c}(\theta)$ and express the evolution as a system $\dot{\theta}=f(\theta, \phi), \dot{\phi}=g(\theta, \phi)$ (where $\phi=\phi_{b}-\phi_{a}$ ). For example, for $K=2$ we have

$$
\rho_{a}=\frac{1}{\sqrt{2}} \frac{\operatorname{sn}(\sqrt{2} \theta, 1 / 2)}{\operatorname{dn}(\sqrt{2} \theta, 1 / 2)}, \quad \rho_{b}=\operatorname{cn}(\sqrt{2} \theta, 1 / 2)
$$

(where sn and cn are Jacobi elliptic functions [3]) and the evolution is given by

$$
\begin{aligned}
& \dot{\theta}=\frac{2}{h^{2}} \sin \phi, \\
& \dot{\phi}=\frac{2}{h^{2}} \frac{\rho_{b}^{2}-\rho_{a}^{2}}{\rho_{a} \rho_{b}} \cos \phi=\frac{4 \sqrt{2}}{h^{2}} \frac{\operatorname{cn}(2 \sqrt{2} \theta, 1 / 2)}{\operatorname{sn}(2 \sqrt{2} \theta, 1 / 2)} \cos \phi .
\end{aligned}
$$

The second conserved quantity (5.15) provides us with the equation of the orbits

$$
\frac{\operatorname{dn}(2 \sqrt{2} \theta, 1 / 2)-1}{\operatorname{sn}(2 \sqrt{2} \theta, 1 / 2)} \cos \phi=\frac{\operatorname{sn}(\sqrt{2} \theta, 1 / 2) \operatorname{cn}(\sqrt{2} \theta, 1 / 2)}{\operatorname{dn}(\sqrt{2} \theta, 1 / 2)} \cos \phi=\sqrt{2} C,
$$

which is represented in Figure 1.

If $\epsilon=+1$ there are additional solutions. The reduced equation (5.11) is equivalent the the following prescription

- A point $n$ in the lattice with $\left|Q_{n}\right|=1$, has neighbours with arbitrary values $Q_{n+1}$, $Q_{n-1}$.

- A point $n$ in the lattice with $\left|Q_{n}\right| \neq 1$ has neighbours with equal values $Q_{n+1}=Q_{n-1}$.

A typical solution is of the form:

$$
Q_{2 n}=e^{i \theta(t)}, \quad Q_{2 n+1}=\rho_{n}(t) e^{i \phi_{n}(t)} .
$$

Substituting into (1.1) we get for $\theta(t), \rho_{n}(t)$ and $\phi_{n}(t)$ the equations

$$
\begin{gathered}
\dot{\theta}=-\frac{2}{h^{2}}, \quad \dot{\rho}_{n}=-\frac{2}{h^{2}}\left(1-\rho_{n}^{2}\right) \sin \left(\theta-\phi_{n}\right), \\
\dot{\phi}_{n}=-\frac{2}{h^{2}}+\frac{2}{h^{2}} \frac{1-\rho_{n}^{2}}{\rho_{n}} \cos \left(\theta-\phi_{n}\right)
\end{gathered}
$$




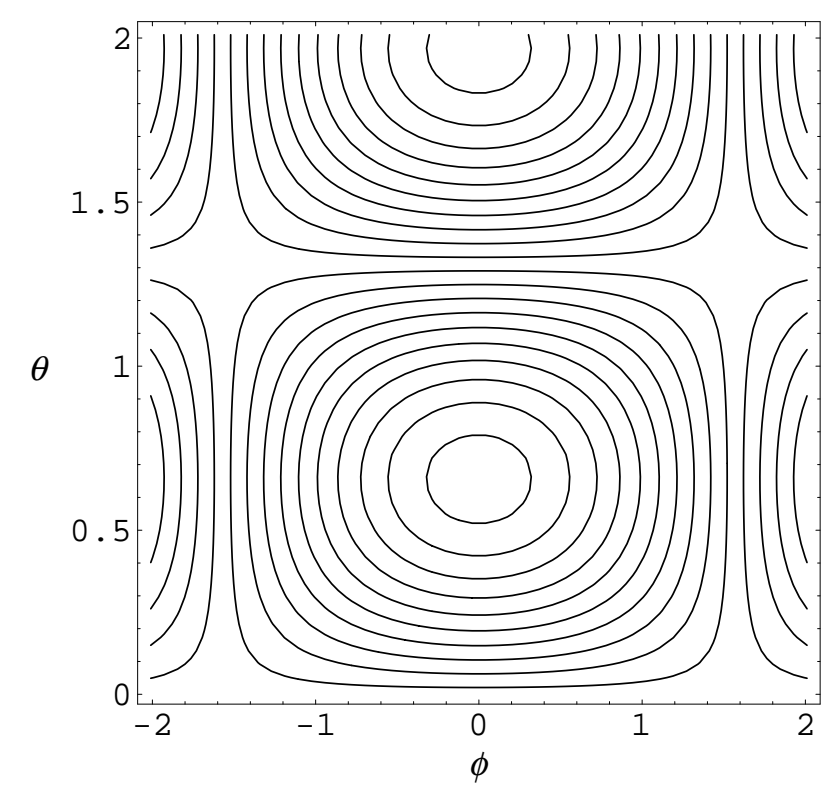

Figure 1. Level curves of (5.16), $\theta$ vs. $\phi$ for $Y_{2}$-reduced dNLS, for $K=2$ and different values of $C$.

Let us notice that in these equations the dependence on $n$ is parametric. It is possible to find the general solution of $(5.17)$, which is given by

$$
\begin{aligned}
& \theta(t)=-\frac{2}{h^{2}} t+\theta_{0}, \\
& \rho_{n}(t)=1+A_{n}+q_{n}^{2}(t), \\
& \sin \phi_{n}(t)=\frac{-q_{n} \cos \theta \pm \sqrt{1+A_{n}} \sin \theta}{1+A_{n}+q_{n}^{2}}
\end{aligned}
$$

where $A_{n}$ is an arbitrary function of $n$, and there are three different expressions for $q_{n}(t)$ depending on the value of $A_{n}$

$$
\begin{array}{ll}
q_{n}^{(1)}(t)=\sqrt{\left|A_{n}\right|} \frac{e^{\frac{4 \sqrt{\left|A_{n}\right|}}{h^{2}}\left(t-t_{n}\right)}+1}{1-e^{\frac{4 \sqrt{\left|A_{n}\right|}}{h^{2}}}\left(t-t_{n}\right)} & \text { if }-1<A_{n}<0 \\
q_{n}^{(2)}(t)=-\frac{h^{2}}{2\left(t-t_{n}\right)} & \text { if } A_{n}=0 \\
q_{n}^{(3)}(t)=\sqrt{\left|A_{n}\right|} \tan \left[\frac{2 A_{n}}{h^{2}}\left(t-t_{n}\right)\right] & \text { if } 0<A_{n}
\end{array}
$$

with $t_{n}$ an arbitrary function of $n$.

The general reduced solution when $\epsilon=+1$ can be described as a piecewise function $Q_{n}$ on the lattice, as it is discussed in [10]. Every piece or "domain" is characterized by a sequence of points with an equal value $Q_{n}=e^{i \theta(t)}$, interspaced with a sequence of points with values $Q_{n}=\rho_{n}(t) e^{i \phi_{n}(t)}$ of modulus and phase given by (5.19), (5.20). At the extremes of each domain we find points where $Q_{n}$ has modulus 1 ; different domains are characterized by a priori different values of the phases. See fig. 2 for an example. 


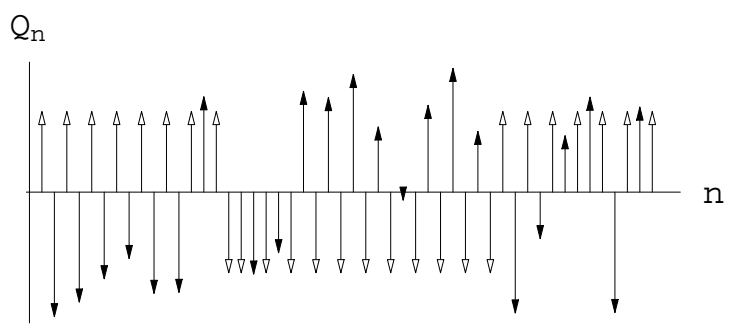

Figure 2. Schematic plot of $Q_{n}$ at a given time $t$, showing three "domains", solution of the $Y_{2}$-reduced dNLS. The white arrowheads correspond to the real values of the points of modulus 1 that define the domains. The black arrowheads correspond to the real values of the points inside a domain of modulus and phase given by eqs. $(5 \cdot 19,5.20)$.

Reduction by $\mathbf{Y}_{\mathbf{3}}$ The reduction is obtained by solving the following equation

$$
\left(1-\epsilon\left|Q_{n}\right|^{2}\right)\left(Q_{n-1}+Q_{n+1}\right)-2 Q_{n}=0 .
$$

Taking into account the dNLS equation (1.1), (5.21) implies $\dot{Q}_{n}=0$. Writing $Q_{n}$ in polar coordinates as

$$
Q_{n}=\rho_{n} \exp \left(i \theta_{n}\right)
$$

we have that

$$
\begin{aligned}
& \left(1-\epsilon \rho_{n}^{2}\right)\left[\rho_{n+1} \sin \left(\theta_{n+1}-\theta_{n}\right)-\rho_{n-1} \sin \left(\theta_{n}-\theta_{n-1}\right)\right]=0 \\
& \left(1-\epsilon \rho_{n}^{2}\right)\left[\rho_{n+1} \cos \left(\theta_{n+1}-\theta_{n}\right)-\rho_{n-1} \cos \left(\theta_{n}-\theta_{n-1}\right)\right]=2 \rho_{n}
\end{aligned}
$$

We can see that $1 \neq \epsilon \rho_{n}^{2}$, so eq.o $(5.23)$ reads

$$
\rho_{n+1} \sin \left(\theta_{n+1}-\theta_{n}\right)=\rho_{n-1} \sin \left(\theta_{n}-\theta_{n-1}\right)
$$

which can be once integrated to get

$$
\sin \left(\theta_{n+1}-\theta_{n}\right)=\frac{K}{\rho_{n+1} \rho_{n}}
$$

where $K$ is an arbitrary integration constant. Substituting (5.26) into (5.24) and taking into account that

$$
\cos \left(\theta_{n+1}-\theta_{n}\right)=\frac{1}{\rho_{n+1} \rho_{n}} \sqrt{\rho_{n+1}^{2} \rho_{n}^{2}-K^{2}}
$$

we get the following difference equation for $\rho_{n}^{2}$

$$
\sqrt{\rho_{n}^{2} \rho_{n+1}^{2}-K^{2}}+\sqrt{\rho_{n}^{2} \rho_{n-1}^{2}-K^{2}}=\frac{2 \rho_{n}^{2}}{1-\epsilon \rho_{n}^{2}} .
$$

Alternatively, substituting $\rho_{n+1}$ and $\rho_{n-1}$ from (5.26) in (5.24) we obtain:

$$
K\left(1-\epsilon \rho_{n}^{2}\right)\left[\operatorname{ctan}\left(\theta_{n+1}-\theta_{n}\right)+\operatorname{ctan}\left(\theta_{n}-\theta_{n-1}\right)\right]=2 \rho_{n}^{2} .
$$


Then

$$
\rho_{n}^{2}=\frac{K\left[\operatorname{ctan}\left(\theta_{n+1}-\theta_{n}\right)+\operatorname{ctan}\left(\theta_{n}-\theta_{n-1}\right)\right]}{2+\epsilon K\left[\operatorname{ctan}\left(\theta_{n+1}-\theta_{n}\right)+\operatorname{ctan}\left(\theta_{n}-\theta_{n-1}\right)\right]}
$$

and substituting (5.29) in (5.25) we find an equation for the phases

$$
\begin{aligned}
& \frac{\operatorname{ctan}\left(\theta_{n+2}-\theta_{n+1}\right)+\operatorname{ctan}\left(\theta_{n+1}-\theta_{n}\right)}{2+\epsilon K\left[\operatorname{ctan}\left(\theta_{n+2}-\theta_{n+1}\right)+\operatorname{ctan}\left(\theta_{n+1}-\theta_{n}\right)\right]} \sin ^{2}\left(\theta_{n+1}-\theta_{n}\right)= \\
& =\frac{\operatorname{ctan}\left(\theta_{n}-\theta_{n-1}\right)+\operatorname{ctan}\left(\theta_{n-1}-\theta_{n-2}\right)}{2+\epsilon K\left[\operatorname{ctan}\left(\theta_{n}-\theta_{n-1}\right)+\operatorname{ctan}\left(\theta_{n-1}-\theta_{n-2}\right)\right]} \sin ^{2}\left(\theta_{n}-\theta_{n-1}\right) .
\end{aligned}
$$

Reduction by $\mathbf{Y}_{4}$ The reduction is obtained by solving

$$
-\frac{t}{h}\left(1-\epsilon\left|Q_{n}\right|^{2}\right)\left(Q_{n+1}-Q_{n-1}\right)+i n h Q_{n}=0
$$

and it can be calculated by a procedure totally analogous to the case of $Y_{3}$. Using again polar coordinates (5.22) we have that the equation for the moduli is

$$
\sqrt{\rho_{n}^{2} \rho_{n+1}^{2}-K(t)^{2}}+\sqrt{\rho_{n}^{2} \rho_{n-1}^{2}-K(t)^{2}}=\frac{n h^{2}}{t} \frac{\rho_{n}^{2}}{1-\epsilon \rho_{n}^{2}}
$$

where $K(t)$ is a first integral

$$
\rho_{n+1} \rho_{n} \cos \left(\theta_{n+1}-\theta_{n}\right)=K(t) .
$$

From the temporal evolution (1.1) given by dNLS it follows that

$$
K(t)=\frac{K_{0}}{t}
$$

and the phases are obtained from the relation

$$
\dot{\theta}_{n}=-\frac{2}{h^{2}}\left[1-\frac{1-\epsilon \rho_{n}^{2}}{\rho_{n}^{2}} K(t)\right] .
$$

\section{Conclusions}

There are several general conclusions to be drawn from this study. The first one, already noticed previously, is that if we wish to study symmetries of difference equations on a fixed and untrasformable lattice and wish to obtain all symmetries that exist in the continuous limit, then generalized symmetries must be considered together with point ones. The linear tools of integrability theory can provide us with the Lie algebra of symmetries $L(h)$ of the considered discrete integrable equation.

The symmetry algebra $L(h)$ includes a very small subalgebra of point transformations. In the continuous limit, when we take the spacing parameter $h$ to 0 , the structure of the Lie algebra changes, and a contraction of Lie algebras occurs, with the lattice spacing $h$ as the contraction parameter. We have already seen this process between the Toda system and the pKdV equation in [16]. If the discrete equation is integrable, $L(h)$ is infinite dimensional, and after the contraction $h \rightarrow 0$ the contracted Lie algebra $L(0)$ is the symmetry 
algebra of the corresponding integrable differential equation, it is still infinite dimensional and includes both generalized and point symmetries. However, a finite-dimensional subset $S \subset L(h)$ of the symmetry algebra of the discrete equation, that includes point and generalized symmetries, contracts into the subalgebra $L_{p} \subset L(0)$ of point symmetries of the corresponding differential equation. In particular we have shown that for dNLS $S$ is a five-dimensional subspace generated by both point and generalized symmetries, that transforms into the five-dimensional point symmetry algebra of the NLS equation. The

subset $S$, though, does not form a Lie algebra, because it is not closed under commutation.

One uses the lowest symmetries to do symmetry reduction of the equation. One thus obtains explicit solutions and discrete analogue of elliptic functions. We have performed several reductions of the discrete NLS equation, obtaining in some cases explicit solutions and in other difference equations of elliptic type for the fields of the lattice. The cases that in the continuous limit, i. e. for the NLS differential equation, produce solvable ordinary differential equations, provide in the discrete non trivial difference equations. We have, though, not been able to consider the case which in the continuous limit gives a Painlevé equation, because in this case the reduced equation contains nonlocal terms.

A question that immediately arises is: how does one find and use symmetries of nonintegrable difference equations, when no linear system is available. One possibility is to give up the notion of a fixed lattice, i. e. study group transformations acting simultaneously on the equations and on the lattice. For instance dilations of coordinates will change the scale of the lattice. This approach has been taken by Dorodnitsyn and collaborators [11], [12]. Another approach is the differential equation method proposed earlier [26]. Here one views a differential-difference equation as an infinite set of differential equations and looks for point symmetries of this infinite system. These will include some, through not necessarily all, generalized symmetries of the differential-difference equation under study. The problem here is that it is often very difficult to solve the corresponding infinite set of determining equations. Finally, let us mention the intrinsic method [26], the one we used in Section 3. It is the simplest to apply and use, and it provides us with the point symmetries of the discrete equation.

\section{Acknowledgments}

We acknowledge support from DGCYT (PB98-0821), the Universidad Complutense / Roma Tre Interchange agreement as well as a NATO Grant (PST. CLG. 978431).

\section{References}

[1] Ablowitz M J and Ladik J F, Nonlinear differential-difference equations J. Math. Phys. 16 (1975), 598-603.

[2] Ablowitz M J and Ladik J F, Nonlinear differential-difference equations and Fourier analysis J. Math. Phys. 17 (1976), 1011-1018.

[3] Abramowitz M and Stegun I A, Handbook of Mathematical Functions, Dover Publications, New York, 1974.

[4] Boiti M and Pempinelli F, Nonlinear Schrödinger equation, Bäcklund transformations and Painlevé transcendents Il Nuovo Cimento 59B (1980), 40-58. 
[5] Bruschi M, Levi D and Ragnisco O, Extension of the Zakharov-Shabat generalized inverse method to solve differential-difference and difference-difference equations $\mathrm{Nuovo} \mathrm{Ci}$ mento 58A (1980), 56-66; Evolution equations associated with the discrete analog of the matrix Schrödinger spectral problem solvable by the inverse spectral transform J. Math. Phys. 22 (1981) 2463-2471.

[6] Bruschi M, Levi D and Ragnisco O, Nuovo Cimento A 48A (1978),213.

[7] Bruschi M and Ragnisco O, Nonlinear differential-difference matrix equations with $n$ dependent coefficients Lett. Nuovo Cimento 31 (1981), 492-496.

[8] Calogero F, Why are certain nonlinear PDEs both widely applicable and Integrable, in: V. E. Zakharov, ed., What is Integrability?, Springer Verlag, New York, 1991, 1-62.

[9] Chiu S C and Ladik J F, Generating exactly soluble nonlinear discrete evolution equations by a generalized Wronskian technique J. Math. Phys. 18 (1977),690-700.

[10] Chubykalo O A, Konotop V V, Vázquez L and Vekslerchik V E, Some features of the repulsive discrete nonlinear Schrödinger equation Phys. Lett. A169 (1992), 359-363.

[11] Dorodnitsyn V A, Finite difference analog of the Noether Theorem Dokl Akad Nauk 328 (1993), 678-681.

[12] Dorodnitsyn V A, Kozlov R and Winternitz P, Lie group classification of second-order ordinary difference equations J. Math. Phys. 41 (2000), 480-504.

[13] Floreanini R and Vinet L, Lie symmetries of finite-difference equations J. Math. Phys. 36 (1995), 7024-7042.

[14] Hernández Heredero R H, Levi D, and Winternitz P, Symmetries of the Discrete Burgers Equation J. Phys. A: Math. Gen. 32 (1999), 2685-2695.

[15] Hernández Heredero R H, Levi D, and Winternitz P, Symmetries of the Discrete Nonlinear Schrödinger Equation, Theor. Math. Phys. 127 (2001), 729-737.

[16] Hernández Heredero R H, Levi D, Rodríguez M A and Winternitz P, Lie algebra contractions and symmetries of the Toda hierarchy J. Phys. A: Math. Gen. 33 (2000), 5025-5040.

[17] Ince E L, Ordinary Differential Equations. Dover Publications, New York, 1956.

[18] Kosevich A M, Kovalev A S, Introduction to nonlinear physical mechanics (Russian) With the collaboration of Yu. S. Kivshar. "Naukova Dumka", Kiev, 1989.

[19] Levi D, Tremblay S and Winternitz P, Lie point symmetries of difference equations and lattices J. Phys. A: Math. Gen.33 ( 2001), 8507-8523.

[20] Levi D and Ragnisco O, Extension of the Spectral - Transform Method for solving Nonlinear Differential Difference Equations Lett. Nuovo Cimento 22 (1978), 691-696.

[21] Levi D and Ragnisco O, Non-linear differential-difference equations with $N$-dependent coefficients II J. Phys. A: Math. Gen. 12 (1979), L163-L167.

[22] Levi D, Ragnisco O and Rodríguez M A, On non-isospectral flows, Painlevé equations and symmetries of differential and difference equations Teor. Mat. Fizika 93 (1992), 473-480.

[23] Levi D and Rodríguez M A, Symmetry group of partial differential equations and of differential difference equations: the Toda lattice versus the Korteweg-de Vries equation J. Phys. A: Math. Gen. 25 (1992), L975-979. 
[24] Levi D and Rodríguez M A, Lie symmetries for integrable evolution equations on the lattice J. Phys. A: Math. Gen. 32 (1999), 8303-8316.

[25] Levi D, Vinet L and Winternitz P, Lie group formalism for difference equations J. Phys. A: Math. Gen. 30 (1997), 633-649.

[26] Levi D and Winternitz P, Continuous symmetries of discrete equations Phys. Lett. A152 (1991), 335-338; Symmetries and conditional symmetries of differential-difference equations J. Math. Phys. 34 (1993), 3713-3730.

[27] Maeda S, The similarity method for difference equations IMA J. Appl. Math. 38 (1987), 129-134; Canonical structure and symmetries for discrete systems Math. Japonica 25 (1980), 405-420.

[28] Quispel G R W, Capel H W and Sahadevan R, Continuous symmetries of differential-difference equations: the Kac-van Moerbeke equation and Painlevé reduction Phys. Lett. A170 (1992), $379-383$.

[29] Quispel G R W, Nijhoff F W, Capel H W and Van der Linden J, Physica 125A (1984), $551-558$.

[30] Vekslerchik V E, An $O(3,1)$ nonlinear $\sigma$-model and the Ablowitz-Ladik hierarchy, J. Phys. A: Math. Gen. 27 (1994), 6299-6313.

[31] Vekslerchik V E, Konotop V V, Discrete nonlinear Schrödinger equation under non-vanishing boundary conditions, Inverse Problems 8 (1992), 889-909. 